\title{
A Case Report of Cervicitis Gonorrhea
}

\author{
Diah Mira Indramaya, Zahruddin Ahmad, Septiana Widyantari \\ Department of Dermatology and Venereology, Faculty of Medicine, Universitas Airlangga/Dr. Soetomo \\ General Academic Teaching Hospital, Surabaya, Indonesia
}

\begin{abstract}
Background: Gonorrhea refers to a set of clinical conditions involving infection with the sexually acquired bacterial pathogen, Neisseria gonorrhoeae. One of the simplest gonococcal infections in women involves mucosal surfaces of the endocervix that cause cervicitis, a frequently asymptomatic inflammatory condition that results in an abnormal mucopurulent discharge and cervical friability. Purpose: To report a case of cervicitis gonorrhea in a female patient whose sexual partner had a hisstory of unprotected promiscuous intercource with multiple sex worker. Case: A 24-year-old female patient presented with increased vaginal discharge for 4 days. Her husband had a history of having sexual intercourse with a sex worker 8 days before. Physical examination revealed mucopurulent exudate in the genitalia and endocervical canal. The Gram staining showed numerous leucocytes and Gram-negative intracellular diplococci, while the result of polymerase chain reaction (PCR) examination for Chlamydia trachomatis was negative. The patient was treated with single-dose cefixime $400 \mathrm{mg}$ orally and doxycycline 100 mg orally twice a day for 7 days. Discussion: The present case report showed cervicitis gonorrhea co-infected with a nonspecific genital infection that was felt asymptomatic and unintentionally found when the patient accompanied her husband to Sexually Transmitted Infection (STI) clinic. The presumptive treatment of cervicitis directed against N. gonorrhoeae and C. trachomatis in this patient leads to a complete cure of the disease. Conclusion: It is important to treat the sexual partner of a patient with urethritis gonorrhea. The empiric treatment of cervicitis that is directed against $N$. gonorrhoeae and also against C. trachomatis is mandatory in preventing further possible complications.
\end{abstract}

Keywords: Sexually Transmitted Infection, gonorrhea, Chlamydia, treatment.

Correspondence address: Diah Mira Indramaya. Department of Dermatology and Venereology, Faculty of Medicine Universitas Airlangga/ Dr. Soetomo General Academic Teaching Hospital, Mayjen Prof. Dr. Moestopo Street No. 6-11 Surabaya 60131. Indonesia. Phone: +623 5501609, email: idiahmira@yahoo.com.

\section{BACKGROUND}

Gonorrhea refers to a set of clinical conditions involving infection with the sexually acquired bacterial pathogen, Neisseria gonorrhoeae, identified microbiologically by its Gram negative intracellular diplococci. ${ }^{1}$ One of the simplest gonococcal infections in women involves mucosal surfaces of the endocervix that cause cervicitis, a frequently asymptomatic, inflammatory condition of the cervix that results in an abnormal mucopurulent discharge and cervical friability. ${ }^{2,3,4}$ Cervicitis is common and considered to arise in rates as a consequence of sexually transmitted infections (STI) and is generally considered to be associated with pathogens mainly $N$. gonorrhoeae and/or Chlamydia trachomatis. At the cervix, those organisms infect the columnar epithelial cells typically located in the endocervix. ${ }^{2,3,5}$ Collectively gonorrheal and chlamydial diseases account for approximately $50 \%$ of the cases of cervicitis. The etiology of the cases of cervicitis not due to gonorrhea or chlamydia remains unclear in several studies. Proposed pathogens include Mycoplasma genitalium, Ureaplasma urealyticum, $U$. parvum, Trichomonas vaginalis, herpes simplex virus (HSV), cytomegalovirus (CMV), adenovirus, and vaginal flora associated with bacterial vaginosis $(\mathrm{BV}){ }^{2,4,6,7}$

In Indonesia, the data from "Survei Terpadu Biologis dan Perilaku (Integrated Biological and Behavioral Survey)" by the Ministry of Health in 2015 reported that infections of gonorrhea and chlamydia were respectively found in $21,20 \%$ and $32,21 \%$ of the population of direct commercial sex workers. So that the men who have sex with them will be at higher risk of getting infected with gonorrhea and chlamydia and then spread the infection to their wives or sexual partners. ${ }^{8}$ A significant percentage of women $>85 \%$ with a urogenital gonococcal infection are asymptomatic so that they will not get examined and treated. ${ }^{9}$

Untreated cervicitis can cause the progression into serious complications such as infertility, ectopic pregnancy, and pelvic inflammatory disease (PID), even in asymptomatic cases. It may also affect sexual health at a population level, as it increases shedding of 
other STIs such as Human Immunodeficiency Virus (HIV) and Human T-cell Lymphotropic Virus (HTLV). Another complication concerning cervicitis caused by $N$. gonorrhoeae is its potential to invade the bloodstream, resulting in gonococcemia and disseminated gonococcal affliction of the joints (especially knees), cardiovascular system, skin, and central nervous system. , $^{3,9,10,11}$

Regarding those complications, Centers for Disease Control (CDC) has recommended presumptive treatment with antimicrobials for C. trachomatis and $N$. gonorrhoeae for women at increased risk (e.g., those aged $<25$ years and those with a new sex partner, a sex partner with concurrent partners, or a sex partner who has a sexually transmitted infection), especially if follow-up cannot be ensured or if testing with nucleic acid amplification test (NAAT) is not possible. ${ }^{6,12}$ The case about cervicitis gonorrhea co-infected with nonspecific genital infection in a woman that felt asymptomatic and unintentionally found when she accompanied her husband to STI clinic was reported. The presumptive treatment of cervicitis had applied in this patient lead to a complete cure of the disease.

\section{CASE REPORTS}

A 24-year-old female patient presented with increased vaginal discharge for 4 days. The discharge was odorless, thick, and slightly yellow. She did not seem aware of the abnormality or any symptoms. There were also no itchy, no fever, no pelvic and abdominal pain, and no pain during micturition. She had already married and had sexual intercourse with her husband 5 days before. She denied having multiple sexual partners. She was unintentionally examined when accompanied by her husband that complained about urethral discharge and got a diagnosis as urethritis gonorrhea at the STI clinic. Her husband had a history of having sexual intercourse with a sex worker 8 days before.

Physical examination revealed mucopurulent exudate (thick yellow pus) in the external genitalia and endocervical canal (figure 1). There was no endocervical bleeding that easily induced by cotton swabs through the cervical ostium. Microscopic examinations for Gram staining and wet preparation of the cervical swab were performed. The Gram staining showed numerous leucocytes ( $>30 /$ high-power field) and Gram-negative intracellular diplococci (figure 2a), and then wet preparation also showed numerous leucocytes without the appearance of $T$. vaginalis and a significant amount of clue cells (figure $2 \mathrm{~b}$ ). The $\mathrm{pH}$ scale of the discharge was 5 , and the whiff test result was negative. The Thayer-Martin culture from endocervix for $N$. gonorrhoeae was positive, while the result polymerase chain reaction (PCR) examination for $C$. trachomatis was negative (figure 3 ). Based on the data, a diagnosis of cervicitis gonorrhea was established.

The patient was treated with a single dose of cefixime $400 \mathrm{mg}$ orally at the first visit and evaluated after 1 week. At the second visit, there were still increased but nonpurulent vaginal discharge and laboratory abnormalities. The Gram staining and wet preparation still showed numerous leucocytes (>30/high-power field), but there were no Gramnegative intracellular diplococci. The diagnosis of nongonococcal and non-chlamydial cervicitis was established. She was prescribed with doxycycline 100 mg orally twice a day for 7 days. At the third visit, the result was good that there was no complaint, no abnormalities of both physical and laboratory examinations.
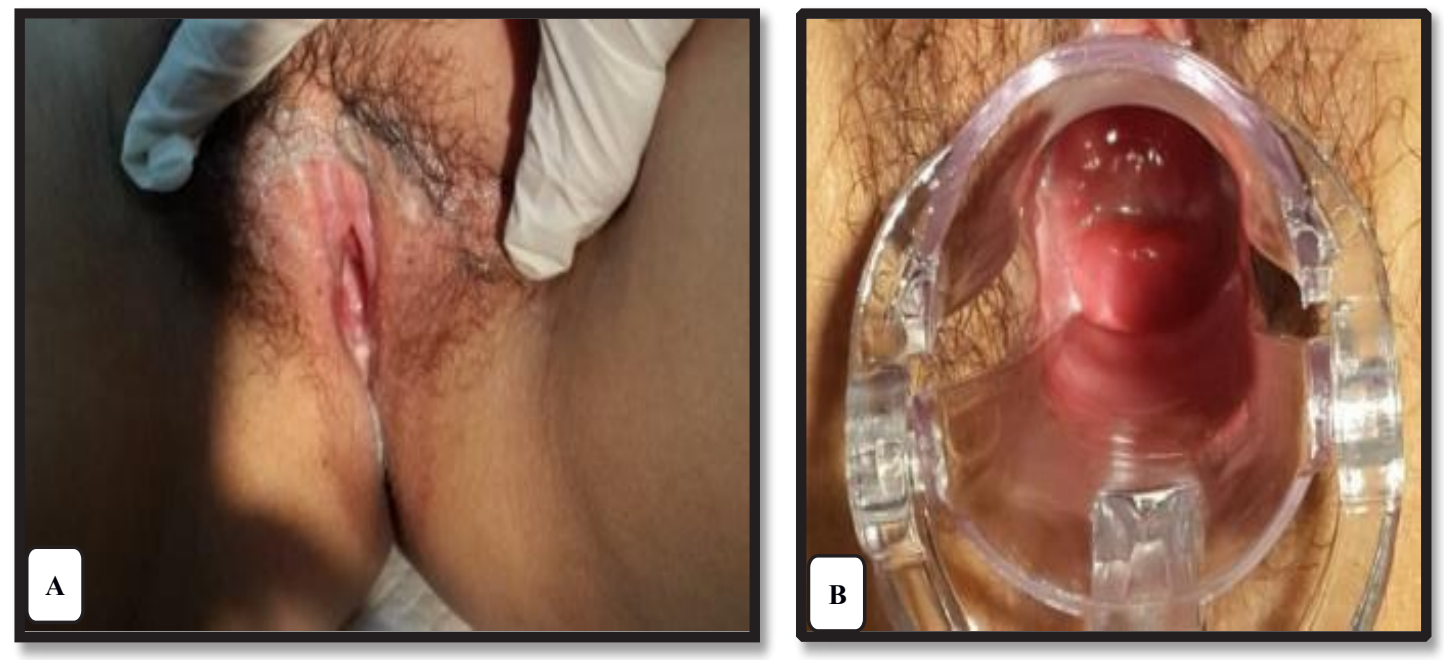

Figure 1. A. Mucopurulent discharge in the external genitalia; B. Mucopurulent endocervical discharge on speculum examination. 

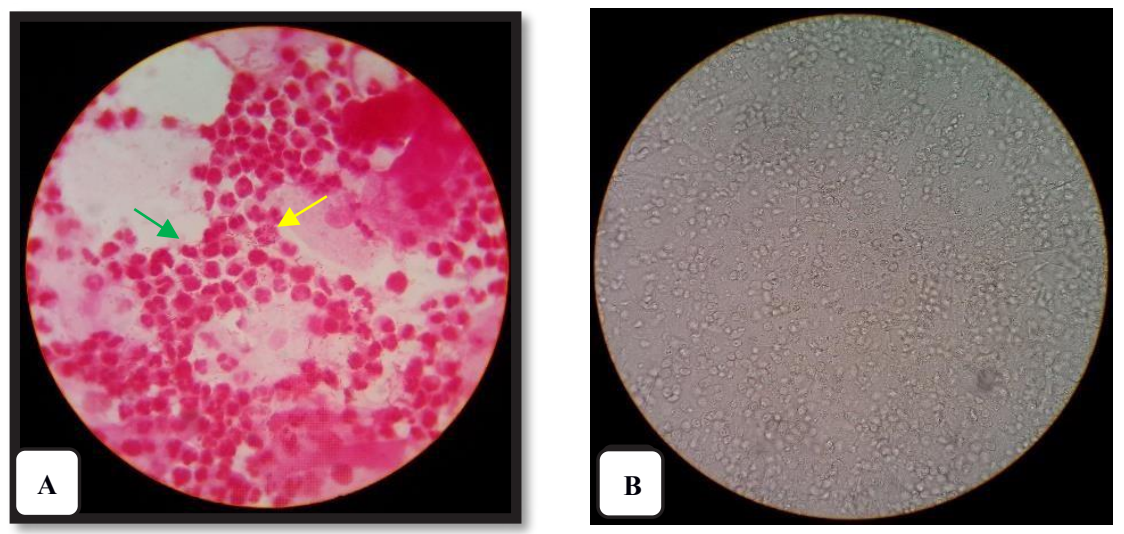

Figure 2. A. Gram staining: Gram-negative intracellular diplococci (yellow arrow) and numerous leucocytes (green arrow); B. Wet preparation: numerous leucocytes.
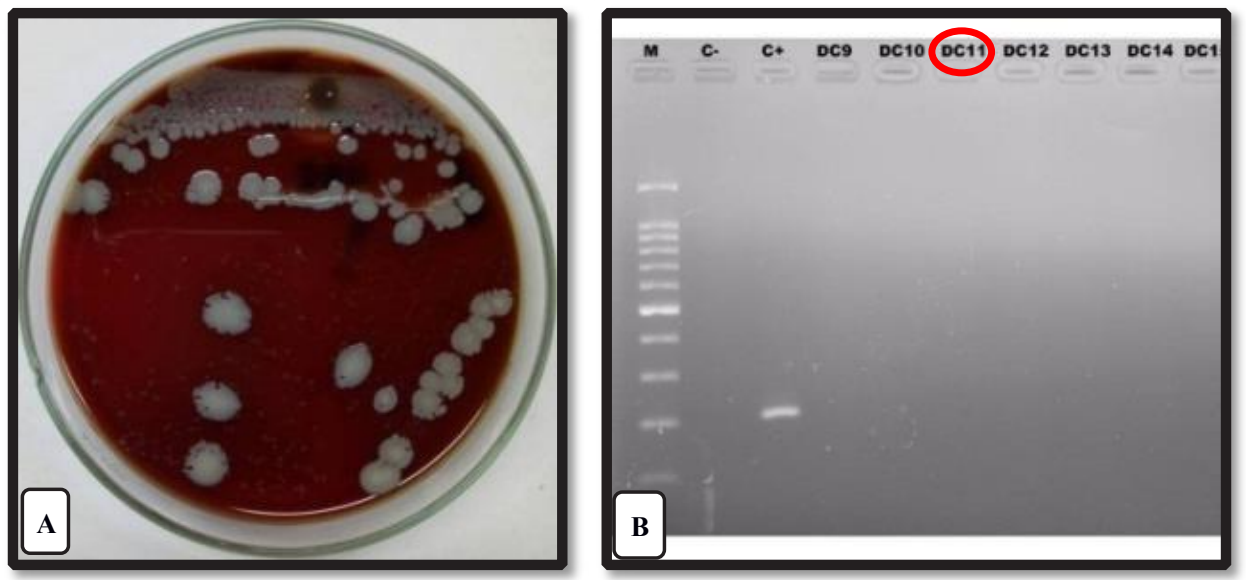

Figure 3. A. Thayer-Martin culture for N. gonorrhoeae; B. PCR for C. trachomatis (C+: positive control; C-: negative control; DC11: our patient)

\section{DISCUSSION}

Cervicitis frequently is asymptomatic, but some women complain of abnormal vaginal discharge and intermenstrual vaginal bleeding (e.g., after sexual intercourse). ${ }^{12}$ It has two main diagnostic hallmarks: a purulent or mucopurulent discharge that is visible in the endocervical canal or on an endocervical smear (generally termed "mucopurulent cervicitis" or simply "cervicitis") and persistent endocervical bleeding, which can be easily induced by the gentle insertion of a swab in the cervix. ${ }^{6}$ The patient, in this case, presented with increased vaginal discharge that was mucopurulent without any other symptoms complained. She had gotten the disease from her husband, who was diagnosed with urethritis gonorrhea. This case demonstrates that patient's sex partner(s) should be referred for evaluation, testing, and treatment. $^{12}$

Cervicitis is diagnosed using a combination of microscopic examination result $\quad>30$ leucocytes/highpower field on microscopic examination) and at least one of the above mentioned clinical signs. In order to detect specific pathogens causing cervicitis, laboratory testing is necessary to identify the organisms involved. Women with cervicitis also should be evaluated for the presence of BV and trichomoniasis, and if these are detected, they should be treated. ${ }^{7,12,13}$ Classically, the organisms most closely recognized to cause cervicitis are $C$. trachomatis and $N$. gonorrhoeae. At the cervix, these organisms infect the columnar epithelial cells typically located in the endocervix. ${ }^{5}$ The other pathogens that can also cause cervicitis include $M$. genitalium, $U$. urealyticum, $U$. parvum, T. vaginalis, HSV, CMV, adenovirus, and vaginal flora associated with $\mathrm{BV} .{ }^{2,4,6,7}$

In this case, the laboratory examinations included Gram staining, wet preparation, $\mathrm{pH}$, whiff test, ThayerMartin culture, and PCR examination for chlamydia. All of those laboratory parameters were needed to find the specific diagnosis and to exclude the differential diagnosis. The Gram staining of urethral and cervical smear is the simplest and the quickest way to diagnose gonorrhea in a routine. In a case of acute gonococcal infection, intracellular Gram-negative diplococci within the neutrophils shall present, as observed in this case. ${ }^{11}$ There were numerous leucocytes and positive 
Thayer-Martin culture to support the establishment of the diagnosis. Trichomoniasis can be excluded since wet preparation did not reveal $T$. vaginalis, while BV can be excluded from the result of the physical and laboratory examinations as it did not meet the Amsel criteria. ${ }^{12}$ PCR examination for chlamydia was performed to ensure the presence of $C$. trachomatis considering that women with gonorrhea are frequently coinfected with chlamydia. ${ }^{1}$

Several factors should affect the decision to provide presumptive therapy for cervicitis. Presumptive treatment with antimicrobials for $C$. trachomatis and $N$. gonorrhoeae should be provided for women at increased risk (e.g., those aged $<25$ years and those with a new sex partner, a sex partner with concurrent partners, or a sex partner who has a sexually transmitted infection), especially if follow-up cannot be ensured or if testing with NAAT is not possible. ${ }^{12,1}$ The patient was prescribed a single dose of cefixime $400 \mathrm{mg}$ orally at the first visit as the diagnosis of cervicitis gonorrhea was established instead of presumptive treatment with double antimicrobials. Furthermore, the patient had been ensured to make the second visit after 1 week, and the PCR chlamydia had been performed to confirm the presence of $C$. trachomatis.

The diagnosis of non-gonococcal and nonchlamydial cervicitis was established based on laboratory examinations at the second visit because there were no Gram-negative intracellular diplococci after cefixime, and the result of the PCR examination for chlamydia was negative. She was prescribed with doxycycline $100 \mathrm{mg}$ orally twice a day for 7 days as evidence-based guidelines recommended azithromycin or doxycycline for cervicitis of unknown etiology. ${ }^{1,2,6,12}$ The result at the third visit was good. There was no abnormality in the physical and laboratory examination so that cervicitis might be caused by another unknown bacteria that was still sensitive to doxycycline. Commonly, women with mucopurulent cervicitis are empirically treated for $C$. trachomatis and $N$. gonorrhoeae. Though associated with cervicitis, $C$. trachomatis and $N$. gonorrhoeae were identified in only half of the cervicitis cases, and causes of the remaining cases were unknown in several studies. ${ }^{7}$

This case had ensured the importance of applying presumptive treatment with double antimicrobials for $C$. trachomatis and $N$. gonorrhoeae in the patient with cervicitis as cervicitis could progress into serious complications such as infertility, ectopic pregnancy, and PID if not promptly treated. ${ }^{10}$ This case demonstrated that patients still need additional treatment with doxycycline after cefixime to eradicate $N$. gonorrhoeae. Evidence has also consistently demonstrated the effects of $C$. trachomatis and $N$. gonorrhoeae as pathogenic bacteria involved in reproductive tract morbidities, including tubal factor infertility and PID. Most cases of tubal factor infertility are attributable to untreated sexually transmitted diseases that ascend along the reproductive tract and are capable of causing tubal inflammation, damage, and scarring. ${ }^{14}$ Women treated for cervicitis should be instructed to abstain from sexual intercourse until they and their partner(s) have been adequately treated (i.e., for 7 days after single-dose therapy or until completion of a 7-day regimen) and symptoms have resolved to minimize transmission and reinfection. ${ }^{12}$

This case highlights the importance of treating sexual partner(s) of a patient with urethritis gonorrhea, although they do not become aware of any symptoms. The examination shall be performed not only for $N$. gonorrhoeae but also for other possible pathogens. The empiric treatment of cervicitis addressing $N$. gonorrhoeae and $C$. trachomatis is important for the management of such patients to prevent further possible complications.

\section{REFERENCES}

1. Walker CK, Sweet RL. Gonorrhea infection in women: prevalence, effects, screening, and management. Int J Womens Health 2011; 3(1): 197-206.

2. Young C, Argaez C. Management and treatment of cervicitis: a review of clinical effectiveness and guidelines. Ottawa: CADTH 2017; 1: 1-21.

3. Pollett S, Calderon M, Heitzinger K, Solari V, Montano SM, Zunt J. Prevalence and predictors of cervicitis in female sex workers in Peru: an observational study. BMC Infect Dis 2013; 13(195): 1-7.

4. Mattson SK, Polk JP, Nyirjesy P. Chronic cervicitis: presenting features and response to therapy. J Low Genit Tract Dis 2016; 20(3): e30e33.

5. Wiesenfeld HC. Much is still unknown about cervicitis of unknown etiology. Sex Transm Dis 2013; 40(5): 386-7.

6. Wagenlehner FME, Brockmeyer NH, Discher T, Friese K, Wichelhaus TA. Klinik, diagnostik und therapie sexuell öbertragbarer infektionen. Dtsch Arztebl Int 2016; 113(1-2): 11-22.

7. Taylor SN, Lensing S, Schwebke J, et al. Prevalence and treatment outcome of cervicitis of unknown etiology. Sex Transm Dis 2014; 40(5): $1-17$.

8. Kementrian Kesehatan Republik Indonesia 2015. Survei terpadu biologis dan perilaku 2015. Jakarta: Kemenkes RI; 2015. p. 23-36. 
9. Morgan MK, Decker CF. Gonorrhea. Dis Mon 2016; 62(8): 260-8.

10. Wilson SP, Vohra T, Knych M, et al. Gonorrhea and chlamydia in the emergency department: continued need for more focused treatment for men, women and pregnant women. Am J Emerg Med 2017; 35(5): 701-3.

11. Skerlev M, Čulav-Košćak I. Gonorrhea: new challenges. Clin Dermatol 2014; 32(2): 275-81.
12. Workowski KA, Bolan GA. Sexually transmitted diseases treatment guidelines, 2015. MMWR Recomm Rep 2015; 64(3): 51-81.

13. Zemouri C, Wi TE, Kiarie J, Seuc A, Mogasale $\mathrm{V}$, Latif A, et al. The performance of the vaginal discharge syndromic management in treating vaginal and cervical infection: a systematic review and meta-analysis. Plos One 2016: 1-21.

14. Tsevat DG, Wiesenfeld HC, Parks C, Peipert JF. Sexually transmitted diseases and infertility. Am J Obstet Gynecol 2017; 216(1): 1-9. 